\title{
ARTICLE OPEN Disease severity dictates SARS-CoV-2-specific neutralizing antibody responses in COVID-19
}

Xiangyu Chen ${ }^{1}$, Zhiwei Pan ${ }^{1}$, Shuai Yue ${ }^{1}$, Fei Yu ${ }^{2}$, Junsong Zhang ${ }^{3}$, Yang Yang ${ }^{1}$, Ren $\mathrm{Li}^{4,5}$, Bingfeng Liu ${ }^{2}$, Xiaofan Yang $^{2}$, Leiqiong Gao ${ }^{1}$, Zhirong Li ${ }^{1}$, Yao Lin ${ }^{1}$, Qizhao Huang ${ }^{6}$, Lifan $\mathrm{Xu}^{1}$, Jianfang Tang ${ }^{1}$, Li Hu${ }^{1}$, Jing Zhao ${ }^{7}$, Pinghuang Liu ${ }^{8}$, Guozhong Zhang ${ }^{9}$, Yaokai Chen $\mathbb{D i D}^{10}$, Kai Deng (D) $^{2,11}$ and Lilin Ye ${ }^{1}$

COVID-19 patients exhibit differential disease severity after SARS-CoV-2 infection. It is currently unknown as to the correlation between the magnitude of neutralizing antibody $(\mathrm{NAb})$ responses and the disease severity in COVID-19 patients. In a cohort of 59 recovered patients with disease severity including severe, moderate, mild, and asymptomatic, we observed the positive correlation between serum neutralizing capacity and disease severity, in particular, the highest NAb capacity in sera from the patients with severe disease, while a lack of ability of asymptomatic patients to mount competent NAbs. Furthermore, the compositions of NAb subtypes were also different between recovered patients with severe symptoms and with mild-tomoderate symptoms. These results reveal the tremendous heterogeneity of SARS-CoV-2-specific NAb responses and their correlations to disease severity, highlighting the needs of future vaccination in COVID-19 patients recovered from asymptomatic or mild illness.

Signal Transduction and Targeted Therapy (2020)5:180

; https://doi.org/10.1038/s41392-020-00301-9

\section{INTRODUCTION}

As of July 28, 2020, the pandemic of coronavirus disease 2019 (COVID-19), caused by severe acute respiratory syndrome coronavirus 2 (SARS-CoV-2) infection, has claimed 16,341,920 clinically confirmed cases and 650,805 deaths worldwide. ${ }^{1}$ The infected patients show heterogeneous clinical manifestations, which can be generally classified into four groups, including severe, moderate, mild, and asymptomatic, according to the severity of symptoms. ${ }^{2}$ Despite daily increasing confirmed cases and death, currently no medical agents are approved to prevent SARS-CoV-2 infection or treat COVID-19 patients.

A growing body of evidence shows that recovered COVID-19 patients can generate immunoglobulin $\mathrm{G}$ (lgG)-type antibodies specifically binding to various structure proteins of SARS-CoV-2 particles shortly after the onset of disease, albeit at variable levels. ${ }^{3-6}$ Among these virus-specific antibodies, only those capable of blocking SARS-CoV-2 spike (S) protein-mediated viral attachment and/or entry of host cells, called neutralizing antibodies (NAbs), can effectively curtail infection. ${ }^{7}$ The convalescent plasma or sera containing NAbs harvested from recovered patients have shown promising results in treating COVID-19 patients of critical illness in several small-scale clinic trials. ${ }^{8-11}$ In addition, a variety of human monoclonal antibodies (mAbs) of potent SARS-CoV-2 neutralizing activities has been cloned from memory B cells from recovered COVID-19 patients, ${ }^{12-21}$ holding great potentials for prophylactic or therapeutic use. However, little is known regarding the relationship between disease severity and the magnitude of SARS-CoV-2-specific NAb responses in patients recovered from COVID-19. Defining the association of disease severity with NAb responses will facilitate the screening of COVID19 recovered patients as therapeutic plasma donors as well as memory B cell providers for cloning high-affinity human neutralizing mAbs to prevent or treat COVID-19.

The circulation of high-titer NAbs provides the immediate protection against corresponding viral infections, which can be achieved by recovering from natural infection or by inducing from vaccine immunization. Thus far, there is no vaccine approved for COVID-19 prophylaxis, albeit several types of COVID-19 vaccines, including inactivated, vector-based, DNA and mRNA vaccines, ${ }^{22-25}$ are undergoing early stages of clinical trials. In addition, the NAb titers can predict the possibility of reinfection in patients recovered from a primary viral infection. Currently, there are few clues regarding whether the patients recovered from COVID-19 can be protected from re-infection or will still require vaccination in the future when effective vaccines become available.

\footnotetext{
${ }^{1}$ Institute of Immunology, PLA, Third Military Medical University, 400038 Chongqing, China; ${ }^{2}$ Institute of Human Virology, Key Laboratory of Tropical Disease Control of Ministry of Education, Zhongshan School of Medicine, Sun Yat-sen University, 510080 Guangzhou, China; ${ }^{3}$ Guangdong Provincial People's Hospital, Guangdong Academy of Medical Sciences, 510080 Guangzhou, China; ${ }^{4}$ State Key Laboratory of Veterinary Biotechnology, Harbin Veterinary Research Institute, Chinese Academy of Agricultural Sciences, 150001 Harbin, Heilongjiang, China; ${ }^{5}$ College of Veterinary Medicine, Northeast Agricultural University, 150030 Harbin, Heilongjiang, China; ${ }^{6}$ Cancer Center, The General Hospital of Western Theater Command, 610083 Chengdu, Sichuan, China; ${ }^{7}$ Biomedical Analysis Center, Third Military Medical University, 400038 Chongqing, China; ${ }^{8}$ Comparative Immunology Research Center, College of Veterinary Medicine, China Agricultural University, 100193 Beijing, China; ${ }^{9}$ Key Laboratory of Animal Epidemiology of the Ministry of Agriculture, College of Veterinary Medicine, China Agricultural University, 100193 Beijing, China; ${ }^{10}$ Chongqing Public Health Medical Center, 400038 Chongqing, China and ${ }^{11}$ Guangzhou Eighth People's Hospital, Guangzhou Medical University, 510050 Guangzhou, China

Correspondence: Guozhong Zhang (zhanggz@cau.edu.cn) or Yaokai Chen (yaokaichen@hotmail.com) or Kai Deng (dengkai6@mail.sysu.edu.cn) or Lilin Ye (yelilinlcmv@tmmu.edu.cn) These authors contributed equally: Xiangyu Chen, Zhiwei Pan, Shuai Yue, Fei Yu, Junsong Zhang
} 


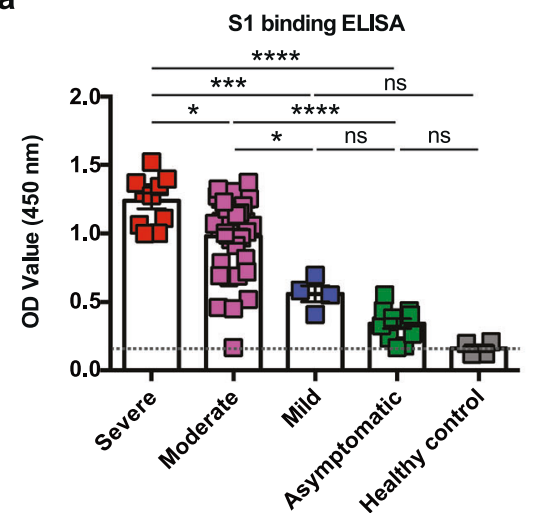

b

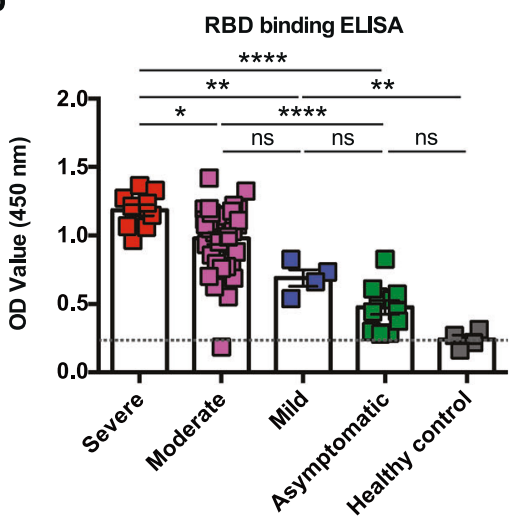

C

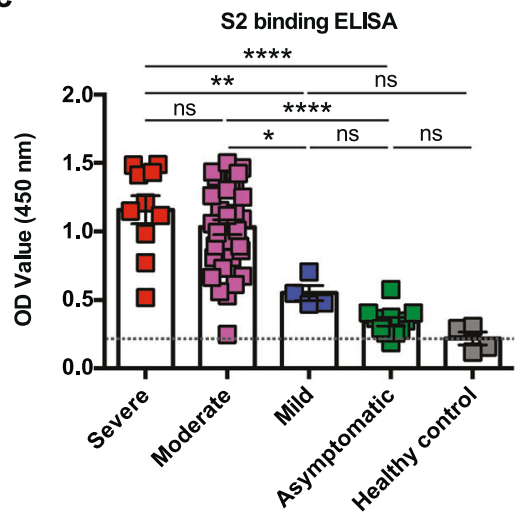

Fig. 1 Antibody responses to SARS-CoV-2 in COVID-19 recovered patients with different symptom severity. a-c ELISA binding assays of 100fold diluted COVID-19 patient sera to ELISA plates after coating with SARS-CoV-2 S1 (a), RBD (b), and S2 (c) proteins. The dashed lines in a-c represent the average values of the healthy control groups. ${ }^{*} P<0.05,{ }^{*} P<0.01,{ }^{* *} P<0.001$, and ${ }^{* * * *} P<0.0001$. ns not significant. Error bars in a-c indicate SEM

\section{RESULTS}

Antibody responses to SARS-CoV-2 in COVID-19 recovered patients with different symptom severity

To explore the potential association between SARS-CoV-2 S protein-specific antibody responses and the disease severity in recovered COVID-19 patients, we included a cohort of 59 adult patients, 48 patients with mild $(n=4)$, moderate $(n=34)$, and severe $(n=10)$ symptoms admitted to Guangzhou Eighth People's Hospital and 11 asymptomatic adult patients admitted to Chongqing Public Health Center Hospital. The median age of these patients was 47 (33-62, interquartile range (IQR)) years; $50.8 \%$ of the patients were female; serum samples were collected at the day of discharge after symptom resolution and SARS-CoV2 nucleotide testing negative twice by reverse transcriptase polymerase chain reaction (RT-PCR). The median time between the onset of symptom to sample collection was 20 (12-30, IQR) days. Asymptomatic patients were identified by screening those with close-contact history to COVID-19 patients and confirmed by SARS-CoV-2 RT-PCR. The disease severity was stratified into asymptomatic, mild, moderate, and severe, according to the national diagnosis and treatment guideline of COVID-19 (Seventh edition) in China (Supplementary Table 1).

The SARS-CoV-2 S protein consists of S1 and S2 subunits. The receptor-binding domain (RBD) within S1 subunit is essential for virus attachment to host cell receptor, human angiotensinconverting enzyme 2 (ACE2), while S2 is critical for virus entry by mediating viral membrane fusion to host cell membrane. ${ }^{26-28}$ Both S1-RBD and S2 represent important potential targets of NAbs. ${ }^{7}$ We first compared the antibodies that specifically bind to S1, RBD, and S2 of SARS-CoV-2 in the sera of COVID-19 recovered patients with different illness severity by lgG enzyme-linked immunosorbent assay (ELISA). Notably, severe and moderate symptomatic patients mounted the most and second robust S1-, S1-RBD-, and S2-specific antibodies, respectively, while mild and asymptomatic patients exhibited significantly lower abundances of S1-, S1-RBD-, and S2-specific antibodies (Fig. 1a-C), highlighting the disease severity as a key determinant factor of the levels of antibodies specific to SARS-CoV-2 S proteins.

Next, we assessed the abilities of antibodies in these COVID-19 recovered patients to block the interaction between RBD and ACE2. Similar to SARS-CoV-2 RBD-binding antibodies, the sera from recovered patients with severe symptoms displayed the highest scores of blocking RBD-ACE2 engagement, followed by patients with moderate symptoms, while antibodies from mild and asymptomatic patients showed much inferior capacity to inhibit RBD-ACE2 interaction (Fig. 2a, b).
NAb responses to SARS-CoV-2 in COVID-19 recovered patients Subsequently, we conducted SARS-CoV-2 S protein pseudotyped lentiviral-based neutralization assay to examine the neutralization capacity of sera from COVID-19 recovered patients. Such assay has been proven to be free of biosafety issue but as reliable as the canonical plaque assay with authentic SARS-CoV-2. ${ }^{17,19,21}$ We observed that the sera neutralization capacity was positively correlated to disease severity (Fig. 2c). Specifically, $80 \%$ of patients with severe symptoms and $47.1 \%$ of patients with moderate symptoms generated antibodies capable of completely neutralizing pseudovirus infection, while only $25 \%$ of serum samples from patients with mild symptoms were able to block pseudovirus infection; strikingly, sera from asymptomatic patients showed no activity to neutralize pseudovirus infection in this assay (Fig. 2d). Next, we performed neutralization assay with the authentic SARSCoV-2 to explore the neutralization capacity of sera from COVID-19 recovered patients. In accordance with the pseudotyped lentiviralbased neutralization assay, we found that the sera neutralization capacity of severe patients was the highest and was 61.1-fold, 1319.1-fold, and 2972.0-fold higher than those of moderate, mild, and asymptomatic patients, respectively (Fig. 2e, f).

Furthermore, the binding abilities of S1, RBD, and S2 were positively correlated to each other (Supplementary Fig. 1a-c) and also positively correlated to both pseudovirus-neutralizing capacities (Supplementary Fig. 1d-f) and authentic SARS-CoV-2 virusneutralizing capacities (Supplementary Fig. 1g-i). These data together suggested that the disease severity determines both the magnitude and neutralizing capacity of SARS-CoV-2-specific antibodies in recovered COVID-19 patients.

Subtypes of NAbs to SARS-CoV-2 S proteins in COVID-19 recovered patients

Given that NAbs can potentially target both S1 and S2 to block viral infection, ${ }^{7}$ we set out to distinguish S1- and S2-specific NAbs in COVID-19 patients with aforementioned pseudovirus neutralization assay. To this end, we used biotin-labeled S1 or S2 recombinant protein to deplete corresponding antibodies in sera from 25 COVID-19 patients that were confirmed to be highly neutralizing in Fig. 2c, e (Supplementary Fig. 2a, b). After depletion, we found that, across all neutralizing sera, $40 \%$ of patients generated both competent S1 and S2 NAbs (i.e., post either S1- or S2-specific antibody depletion, sera can still completely neutralize pseudoviruses; labeled as "S1/S2-NAbs"); while $40 \%$ of patients only generated S1-competent NAbs ("S1NAbs only"), and $4 \%$ of patients only generated S2-specific NAbs ("S2-NAbs only"); interestingly, $16 \%$ of serum samples strictly 
a

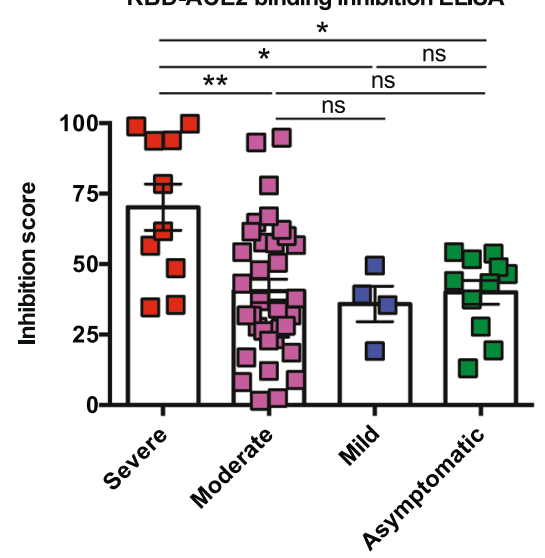

C

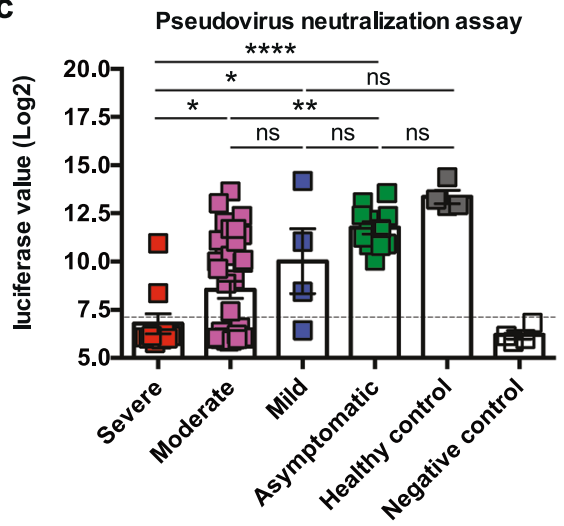

e

SARS-CoV-2 neutralization assay

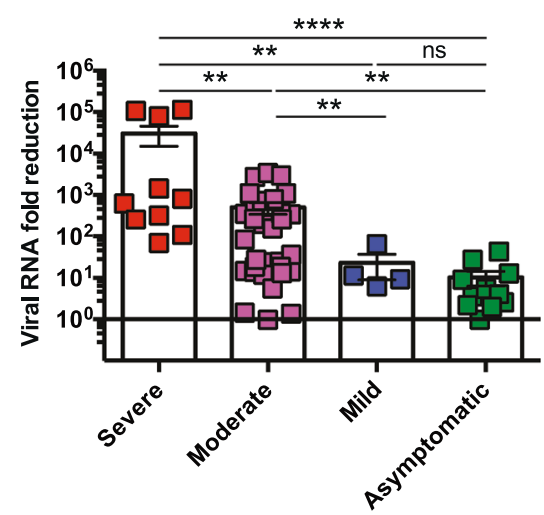

b

RBD-ACE2 binding inhibition score high $(>50)$ and low $(<50)$
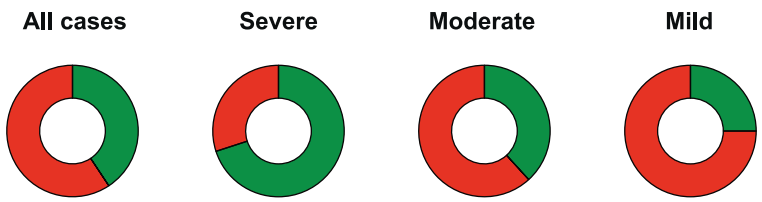

Asymptomatic

$40.7 \% \mid 59.3 \%$

$70.0 \% \mid 30.0 \%$

$38.2 \% \mid 61.8 \%$

$25.0 \% \mid 75.0 \%$

$27.3 \% \mid 72.7 \%$

d

Pseudovirus neutralization positive (<cutoff value) and negative (>cutoff value)

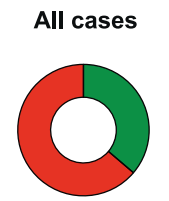

$36.2 \% \mid 63.8 \%$

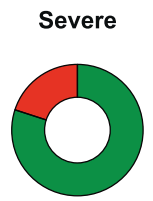

$80.0 \% \mid 20.0 \%$

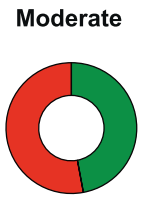

$47.1 \%$ | $52.9 \%$

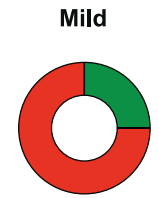

$25.0 \% \mid 75.0 \%$
Asymptomatic

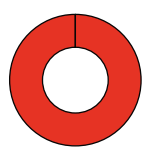

$0 \% \mid 100.0 \%$

f

Fold change between indicated two groups in Fig.2e

\begin{tabular}{ccccc}
\hline & Severe & Moderate & Mild & Asymptomatic \\
\hline Severe & $/$ & 61.1 & 1319.1 & 2972.0 \\
Moderate & 61.1 & $/$ & 21.6 & 48.6 \\
Mild & 1319.1 & 21.6 & $/$ & 2.3 \\
Asymptomatic & 2972.0 & 48.6 & 2.3 & $/$ \\
\hline
\end{tabular}

Fig. 2 Neutralizing antibody responses to SARS-CoV-2 in COVID-19 recovered patients. a Scores showing the COVID-19 patient serummediated inhibition of the SARS-CoV-2 RBD protein binding to ACE2 protein by ELISA. $\mathbf{b}$ Pie charts showing the proportions of patients with high ( $>50$, green) or low ( $<50$, red) RBD-ACE2-binding inhibition score in each indicated situations. c Patient serum-mediated blocking of luciferase-encoding SARS-CoV-2-typed pseudovirus into ACE2/293T cells. The dashed line indicates the cutoff value (7.121) determined by the ROC curve analysis. d Pie charts showing the proportions of patients with pseudovirus neutralization positive $(<7.121$, green) or negative $(>7.121$, red) in each indicated situations. e Patient serum-mediated blocking of SARS-CoV-2 virus into Vero E6 cells. $f$ A table showing the fold change of SARS-CoV-2 viral RNA fold reduction between indicated two groups in e. ${ }^{*} P<0.05,{ }^{* *} P<0.01$, and ${ }^{* * *} P<0.0001$. ns not significant. Error bars in a, c, e indicate SEM

depended on the collaboration of S1- and S2-specific NAbs to effectively neutralize pseudovirus infection (i.e., either S1- or S2specific antibody depletion in the serum can result in the failure of neutralization; labeled as "(S1+S2)-NAbs") (Fig. 3a, b). Among NAbs in severe symptomatic patients, the majority of sera (62.5\%) potently neutralized both $\mathrm{S} 1$-mediated receptor attachment and S2-mediated membrane fusion, while $37.5 \%$ only blocked S1mediated receptor engagement (Fig. 3c). For mild-to-moderate symptomatic patients, NAb features were more diverse: $41.2 \%$ of them consisted of only S1-neutralizing NAbs, $29.4 \%$ possessed the abilities to block both receptor engagement and membrane fusion. Notably, $23.5 \%$ of these sera required the combination of S1- and S2-specific NAbs to effectively neutralize pseudovirus infection (Fig. 3c). Collectively, our data revealed the highly heterogeneous nature of $\mathrm{NAb}$ responses against SARS-CoV-2 S protein and such diversity seemed to be closely associated with 
a

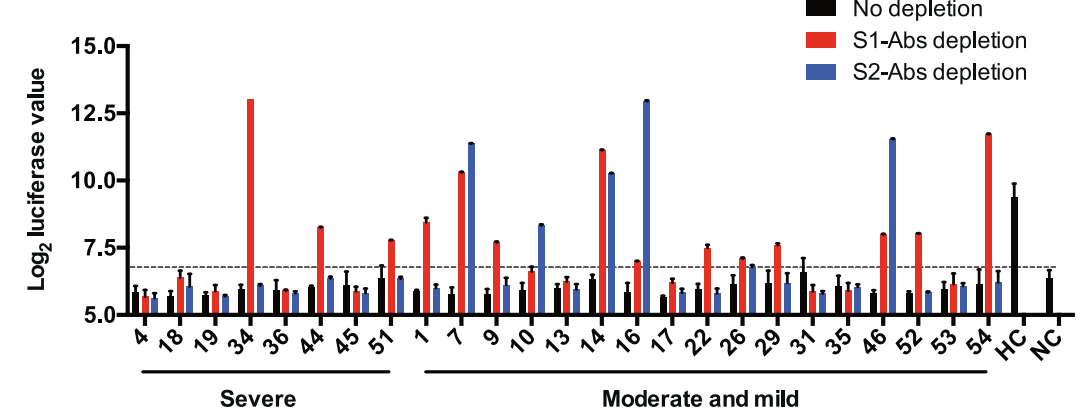

C

Severe $(n=8)$

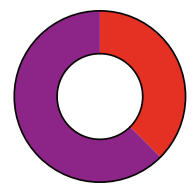

$37.5 \%$, S1-NAbs

$0 \%$, S2-NAbs

$0 \%,(\mathrm{~S} 1+\mathrm{S} 2)$-NAbs

$62.5 \%, \mathrm{~S} 1 / \mathrm{S} 2-\mathrm{NAbs}$
Moderate and mild

Moderate and mild $(n=17)$

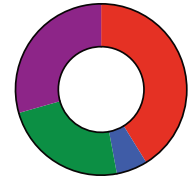

$41.2 \%$, S1-NAbs

$5.9 \%$, S2-NAbs

$23.5 \%,(\mathrm{~S} 1+\mathrm{S} 2)$-NAbs

$29.4 \%$, S $1 /$ S2-NAbs b

Patients of pseudovirus neutralization-positive $(n=25)$

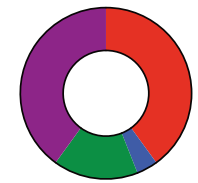

$40.0 \%$, S1-NAbs only $4.0 \%$, S2-NAbs only $16.0 \%$, (S1+S2)-NAbs $40.0 \%, \mathrm{~S} 1 / \mathrm{S} 2-\mathrm{NAbs}$ Patients of S1-nAbs only $(n=10)$

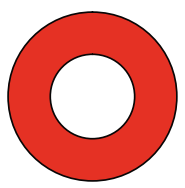

$100.0 \%$ :

RBD-NAbs-dependent $0.0 \%$ : RBD-NAbs-independent

d

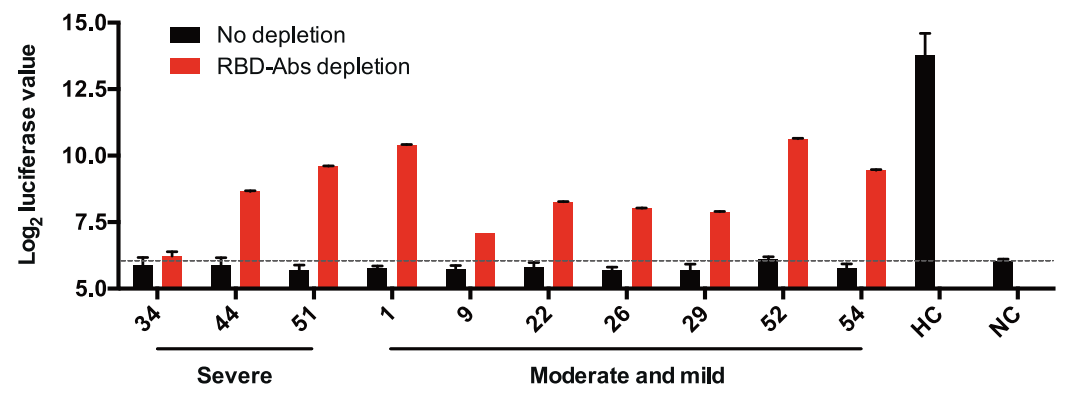

Fig. 3 Subtypes of neutralizing antibodies to SARS-CoV-2 S proteins in COVID-19 recovered patients. a Blocking of luciferase-encoding SARSCoV-2 typed pseudovirus into ACE2/293T cells by patient sera (no depletion) or S1 antibody-depleted sera (S1-Abs depletion) or S2 antibodydepleted sera (S2-Abs depletion). The dashed line indicates the cutoff value (6.749) determined by the ROC curve analysis. HC healthy control, NC negative control. b, c Pie charts showing the proportions of patients with different neutralizing antibody (NAb) subtype responses in the total 25 patients (b), 8 severe patients (c, left panel), and 17 moderate and mild patients (c, right panel) of pseudovirus neutralization positive. d Blocking of luciferase-encoding SARS-CoV-2 typed pseudovirus into ACE2/293T cells by "S1-NAbs only" patient sera with RBD antibody depletion (RBD-Abs depletion) or without RBD antibody depletion (no depletion). The dashed line indicates the cutoff value (6.034) determined by the ROC curve analysis. HC healthy control, NC negative control. e Pie chart showing the proportions of "S1-NAbs only" patients with RBD-Nab-dependent or -independent antibody response. Error bars in a, d indicate SEM

disease severity. The immune mechanisms underlying the diversity of NAbs responses in COVID-19 patients with different degree of symptoms warrant further investigations.

Finally, we investigated whether NAbs depleted by S1recombinant protein are actually targeting RBD for their neutralizing capacity. For this purpose, we depleted RBD-specific antibodies in 10 serum samples showing S1-specific neutralization by biotin-conjugated RBD protein-mediated pull-down (Supplementary Fig. 2c). Antibodies post RBD depletion were shown to lose RBD-binding ability but still keep their binding to both $\mathrm{S} 1$ and S2 proteins, suggesting the efficiency and specificity of RBD Ab depletion (Supplementary Fig. 2c-e). Notably, all sera with S1specific neutralization failed to neutralize pseudovirus infection after RBD-specific NAb depletion (Fig. 3d, e), demonstrating the strict dependency of RBD-specific NAbs to disengage viral attachment to the host receptor. These data provided the rationale for exclusively using RBD as S1-immunogen in vaccine design, in particular, given that several reports have shown the enhanced disease after whole S1 immunization. ${ }^{29,30}$

\section{DISCUSSION}

The COVID-19 patients show stratified symptoms, including asymptomatic, mild, moderate, and severe. ${ }^{2}$ Using RBD-ACE2 blockade, pseudovirus neutralization, and authentic virus neutralization, we observed that disease severity positively correlates to $\mathrm{NAb}$ responses. The patients recovered from severe illness mounted the most robust NAb responses. Strikingly, asymptomatic patients fail to generate competent NAbs. The mechanisms underlying disease severity-associated NAb responses are elusive. One possible explanation is that the induction of SARS-CoV-2specific $\mathrm{NAb}$ responses requires the strengthened and prolonged $B$ cell receptor $(B C R)$ stimulation. Indeed, enhanced BCR rearrangement was observed in COVID-19 patients with severe disease symptom. ${ }^{31}$ This may provide important insights into the COVID19 vaccine design, in which the vaccine regimens should release enough SARS-CoV-2 immunogens in an extended period.

Given the critical role of NAbs in protecting viral infection in airways, the recovered asymptomatic patients may suffer from SARS-CoV-2 re-infection. In this circumstance, these patients need 
to be vaccinated whenever the effective vaccines are available. Thus far, it is unknown as to the protective immunity that prevents asymptomatic patients from progressing to more severe disease. Probably, these patients can mount robust SARS-CoV-2-specific $\mathrm{CD}^{+} \mathrm{T}$ cell responses, which may confer the protection by directly clearing virus-infected target cells. However, this hypothesis needs to be confirmed in future investigations.

Our results also demonstrated the tremendous heterogeneous NAb responses in patients capable of inducing high-titer NAbs. The majority ( $80.7 \%)$ of patients can produce S1-specfic NAbs, and half of these patients are able to generate S2-specific NAbs. However, only around $40 \%$ of patients generated both S1- and S2-specific competent NAbs. Particularly, approximately $7 \%$ patients had to depend on the collaboration between S1- and S2-specific antibodies for efficient viral neutralization. The mechanisms underlying the heterogeneous NAb responses in recovered patients remain unknown and warrant further studies. Notably, all S1-specific NAbs were strictly RBD dependent and deletion of RBDspecific antibodies led to failure in neutralization in S1-specific sera. These results highlighted the importance of S1 RBD itself, but not other parts of S1 protein, in inducing competent NAbs.

In conclusion, we have demonstrated the positive correlation between the magnitude of NAb responses and disease severity in patients recovered from COVID-19. We have also found that disease severity also influences the neutralization heterogeneity of SARS-CoV2-specific antibodies. Our results highlight the needs to include mild illness and asymptomatic patients for future vaccination and also suggest that the collection of plasma from COVID-19 recovered patients should be restricted to those with moderate to severe symptoms for passive antibody therapy. Our data also provide important rationale for exclusively using SARSCoV-2 RBD as S1-immunogen in COVID-19 vaccine regime.

\section{MATERIALS AND METHODS}

Human samples

The 59 COVID-19 recovered patients enrolled in the study provided written informed consent and were from different sources. The sera of the severe, moderate, and mild patients were obtained from Guangzhou Eighth People's Hospital. The sera of the asymptomatic patients were obtained from Chongqing Public Health Medical Center. Healthy control subjects were four adult participants in the study. The study received Institutional Review Board approvals at Guangzhou Eighth People's Hospital (KE202001134) and Chongqing Public Health Medical Center (2020-023-01-KY).

Enzyme-linked immunosorbent assay

As previously described, ${ }^{15} 50 \mathrm{ng}$ of SARS-CoV-2 S1 protein (Sino Biological, 40591-V08H) or SARS-CoV-2 RBD protein (Sino Biological, 40592-V08B) or SARS-CoV-2 S2 protein (Sino Biological, 40590V08B) in $100 \mu \mathrm{l}$ phosphate-buffered saline (PBS) per well was coated on ELISA plates (Costar, 42592) overnight at $4{ }^{\circ} \mathrm{C}$. The ELISA plates were blocked for $1 \mathrm{~h}$ with $100 \mu \mathrm{l}$ blocking buffer (5\% fetal bovine serum (FBS) and $0.1 \%$ Tween 20 in PBS) and then incubated with diluted patient or healthy control sera in $100 \mu \mathrm{l}$ blocking buffer for $1 \mathrm{~h}$. After washing with PBST buffer $(0.1 \%$ Tween 20 in PBS), the ELISA plates were incubated with antihuman IgG horseradish peroxidase (HRP) antibody (Bioss Biotech, 0297D) for $45 \mathrm{~min}$, followed by PBST washing and addition of 3,3',5,5'-tetramethylbenzidine (TMB) buffer (Beyotime, P0209). The ELISA plates were allowed to react for 5-10 min and stopped by $1 \mathrm{M} \mathrm{HCl}$ stop buffer. The optical density (OD) value was detected at $450 \mathrm{~nm}$.

ELISA-based RBD-ACE2-binding inhibition assay As previously described, ${ }^{15} 200 \mathrm{ng}$ of ACE2 protein (Sino Biological, $10108-\mathrm{H} 08 \mathrm{H})$ in $100 \mu \mathrm{l}$ PBS per well was coated on ELISA plates overnight at $4{ }^{\circ} \mathrm{C}$. The ELISA plates were blocked for $1 \mathrm{~h}$ with $100 \mu \mathrm{l}$ blocking buffer (5\% FBS and 0.1\% Tween 20 in PBS); meanwhile, $50 \mu \mathrm{l} 10$-fold diluted patient or healthy control sera were incubated with $7.5 \mathrm{ng}$ SARS-CoV-2 RBD-mouse FC protein (Sino Biological, 40592-V05H) in $50 \mu \mathrm{l}$ blocking buffer for $1 \mathrm{~h}$. Then the incubated sera/SARS-CoV-2 RBD-mouse FC protein mixture was added into the ELISA plates and allowed to develop for $30 \mathrm{~min}$, followed by PBST washing and incubation with anti-mouse FC HRP antibody (Thermo Fisher Scientific, A16084) for $30 \mathrm{~min}$. Next, the ELISA plates were washed with PBST and treated with TMB buffer (Beyotime, P0209). After $5 \mathrm{~min}$, the ELISA reaction was stopped by $1 \mathrm{M} \mathrm{HCl}$ stop buffer and determined at $450 \mathrm{~nm}$. The RBD-ACE2binding inhibition score was calculated as: $100 \times(1-(\mathrm{OD} 450$ value of patient sera/OD450 value of healthy control sera)).

\section{Pseudovirus neutralization assay}

The pseudovirus neutralization assay was previously described. 15,32 Briefly, HEK-293T cells were transfected with pLenti-luciferase, psPAX2, and 2019-nCov S plasmids by using TransIT-293 Transfection reagent (Mirus, MIR 2700). After $12 \mathrm{~h}$, the culture media was changed to fresh media. And at $64 \mathrm{~h}$ after transfection, the culture supernatants containing SARS-CoV-2 typed pseudovirus were harvested. Next, 200-fold diluted patient or healthy control sera were mixed with SARS-CoV-2 typed pseudovirus for $1 \mathrm{~h}$ at $37^{\circ} \mathrm{C}$. Then the ACE2-expressing HEK-293T (ACE2/293T) cells were incubated with the sera/pseudovirus mixture overnight and then cultured with fresh media. At $40 \mathrm{~h}$ after the mixture incubation, the luciferase activity of SARS-CoV-2 typed pseudovirus-infected ACE2/ 293T cells were measured by a luciferase reporter assay kit (Promega, E1910).

\section{SARS-CoV-2 serum neutralization assay}

Patient sera were diluted in Dulbecco's Modified Eagle Medium (40 fold-dilution) and mixed with an equal volume of $80-100$ plaqueforming unit SARS-CoV-2 (EPI_ISL_444969) for $1 \mathrm{~h}$ at $37^{\circ} \mathrm{C}$. Serum-virus mixture were then added to the Vero E6 cell monolayers in 48 -well plates and incubated at $37^{\circ} \mathrm{C}$ in $5 \% \mathrm{CO}_{2}$ for $1 \mathrm{~h}$. After removing the inocula, plates were overlaid with culture medium and cultured at $37{ }^{\circ} \mathrm{C}$ for $48 \mathrm{~h}$. Subsequently, viral RNA from the cultural supernatants was extracted and the viral RNA copies were determined by quantitative PCR according to the viral detection kit's protocol (DAAN Gene Co., Ltd. of Sun Yat-Sen University). All experiments related to authentic viruses were performed in the certified BSL-3 facility of Sun Yat-sen University. The SARS-CoV-2 viral RNA fold reduction $=2^{\text {(CT value of sample }-C T \text { value of mock) }}$.

Depletion of SARS-CoV-2 S protein-specific antibodies

First, SARS-CoV-2 S1 protein (Sino Biological, 40591-V08H) or SARS-CoV-2 RBD protein (Sino Biological, 40592-V08B) or SARSCoV-2 S2 protein was conjugated with biotin by following the manufacturer's protocol (Thermo Fisher Scientific, A39257). Then biotin-conjugated proteins were incubated with BeaverBeads Mag Streptavidin Matrix (Beaver, 22305) at $4{ }^{\circ} \mathrm{C}$ for $1.5 \mathrm{~h}$. After washing with PBS, the SARS-CoV-2 S protein-coupled beads were next incubated with diluted patient sera at $4^{\circ} \mathrm{C}$ for $1.5 \mathrm{~h}$. Then the supernatants were harvested and quality controlled by ELISA assays for SARS-CoV-2 S proteins.

Statistics

The SARS-CoV-2 antibody titers or virus-neutralizing function of the sera belonging to patients with different severity were compared with the one-way analysis of variance test (Tukey's multiple comparisons test). The cutoff value in each pseudovirusneutralizing function assay was determined by the receiver operating characteristic curve analysis and was of the highest likelihood ratio. Correlations between different SARS-CoV-2 antibody titers or between SARS-CoV-2 antibody titers and pseudovirus titers or between SARS-CoV-2 antibody titers and SARS-CoV-2 
virus titers were analyzed using Pearson's correlation coefficient. $P$ values $<0.05$ were defined as statistically significant. Prism 6 software was used for statistical analysis.

\section{DATA AVAILABILITY}

The data sets of the study are available from the corresponding authors upon reasonable request.

\section{ACKNOWLEDGEMENTS}

This work was supported by grants from the National Science and Technology Major Project (No. 2017ZX10202102-006-002 to L.Y.), the National Natural Science Fund for Distinguished Young Scholars (No. 31825011 to L.Y.), and the National Science and Technology Major Project (2016ZX09J16105-001-002 to L.Y.).

\section{AUTHOR CONTRIBUTIONS}

X.C., Z.P., S.Y., F.Y., J. Zhang, Y.Y., R.L., B.L., X.Y., L.G., Z.L., Y.L., Q.H., L.X., J.T., L.H., and J. Zhao performed the experiments. L.Y. designed the study, analyzed the data, and wrote the paper with X.C., X.Z., P.L., Y.W., and K.D.; G.Z., Y.C., K.D. and L.Y. supervised the study.

\section{ADDITIONAL INFORMATION}

The online version of this article (https://doi.org/10.1038/s41392-020-00301-9) contains supplementary material, which is available to authorized users.

Competing interests: The authors declare no competing interests.

\section{REFERENCES}

1. WHO. Coronavirus Disease (COVID-19): Situation Report-190 (WHO, 2020).

2. Wu, Z. \& McGoogan, J. M. Characteristics of and important lessons from the coronavirus disease 2019 (COVID-19) outbreak in China: summary of a report of 72314 cases from the Chinese Center for Disease Control and Prevention. JAMA. https://doi.org/10.1001/jama.2020.2648 (2020).

3. Long, Q. X. et al. Antibody responses to SARS-CoV-2 in patients with COVID-19. Nat. Med. https://doi.org/10.1038/s41591-020-0897-1 (2020).

4. Amanat, F. et al. A serological assay to detect SARS-CoV-2 seroconversion in humans. Nat. Med. https://doi.org/10.1038/s41591-020-0913-5 (2020).

5. Wu, F. et al. Neutralizing antibody responses to SARS-CoV-2 in a COVID-19 recovered patient cohort and their implications. Preprint at https://doi.org/ 10.1101/2020.03.30.20047365 (2020).

6. Ni, L. et al. Detection of SARS-CoV-2-specific humoral and cellular immunity in COVID-19 convalescent individuals. Immunity. https://doi.org/10.1016/j. immuni.2020.04.023 (2020).

7. Huang, A. T. et al. A systematic review of antibody mediated immunity to coronaviruses: antibody kinetics, correlates of protection, and association of antibody responses with severity of disease. Preprint at https://doi.org/10.1101/ 2020.04.14.20065771 (2020).

8. Liu, S. T. H. et al. Convalescent plasma treatment of severe COVID-19: a matched control study. Preprint at https://doi.org/10.1101/2020.05.20.20102236 (2020).

9. Shen, C. et al. Treatment of 5 critically ill patients with COVID-19 with convalescent plasma. JAMA. https://doi.org/10.1001/jama.2020.4783 (2020).

10. Salazar, E. et al. Treatment of Coronavirus Disease (COVID-19) patients with convalescent plasma. Am. J. Pathol. https://doi.org/10.1016/j.ajpath.2020.05.014 (2020).

11. Duan, K. et al. Effectiveness of convalescent plasma therapy in severe COVID-19 patients. Proc. Natl Acad. Sci. USA 117, 9490-9496 (2020).

12. Baum, A. et al. Antibody cocktail to SARS-CoV-2 spike protein prevents rapid mutational escape seen with individual antibodies. Science. https://doi.org/ 10.1126/science.abd0831 (2020).
13. Brouwer, P. J. M. et al. Potent neutralizing antibodies from COVID-19 patients define multiple targets of vulnerability. Science. https://doi.org/10.1126/science. abc5902 (2020).

14. Cao, Y. et al. Potent neutralizing antibodies against SARS-CoV-2 identified by high-throughput single-cell sequencing of convalescent patients $B$ cells. Cell https://doi.org/10.1016/j.cell.2020.05.025 (2020).

15. Chen, X. et al. Human monoclonal antibodies block the binding of SARS-CoV-2 spike protein to angiotensin converting enzyme 2 receptor. Cell. Mol. Immunol. 17, 647-649 (2020).

16. Hansen, J. et al. Studies in humanized mice and convalescent humans yield a SARS-CoV-2 antibody cocktail. Science. https://doi.org/10.1126/science.abd0827 (2020).

17. Ju, B. et al. Human neutralizing antibodies elicited by SARS-CoV-2 infection. Nature. https://doi.org/10.1038/s41586-020-2380-z (2020).

18. Rogers, T. F. et al. Isolation of potent SARS-CoV-2 neutralizing antibodies and protection from disease in a small animal model. Science. https://doi.org/10.1126/ science.abc7520 (2020).

19. Shi, R. et al. A human neutralizing antibody targets the receptor binding site of SARS-CoV-2. Nature. https://doi.org/10.1038/s41586-020-2381-y (2020).

20. Wec, A. Z. et al. Broad neutralization of SARS-related viruses by human monoclonal antibodies. Science. https://doi.org/10.1126/science.abc7424 (2020).

21. Wan, J. et al. Human IgG neutralizing monoclonal antibodies block SARS-CoV-2 infection. Cell Rep. 32, 107918 (2020).

22. Gao, Q. et al. Rapid development of an inactivated vaccine candidate for SARSCoV-2. Science. https://doi.org/10.1126/science.abc1932 (2020).

23. $\mathrm{Yu}$, J. et al. DNA vaccine protection against SARS-CoV-2 in rhesus macaques. Science. https://doi.org/10.1126/science.abc6284 (2020).

24. Zhu, F. C. et al. Safety, tolerability, and immunogenicity of a recombinant adenovirus type-5 vectored COVID-19 vaccine: a dose-escalation, open-label, nonrandomised, first-in-human trial. Lancet. https://doi.org/10.1016/S0140-6736(20) 31208-3 (2020).

25. Amanat, F. \& Krammer, F. SARS-CoV-2 vaccines: status report. Immunity 52, 583-589 (2020).

26. Wrapp, D. et al. Cryo-EM structure of the 2019-nCoV spike in the prefusion conformation. Science 367, 1260-1263 (2020).

27. Yan, R. et al. Structural basis for the recognition of SARS-CoV-2 by full-length human ACE2. Science 367, 1444-1448 (2020).

28. Kirchdoerfer, R. N. et al. Pre-fusion structure of a human coronavirus spike protein. Nature 531, 118-121 (2016).

29. Liu, L. et al. Anti-spike IgG causes severe acute lung injury by skewing macrophage responses during acute SARS-CoV infection. JCI Insight. https://doi.org/ 10.1172/jci.insight.123158 (2019).

30. Du, L. et al. The spike protein of SARS-CoV-a target for vaccine and therapeutic development. Nat. Rev. Microbiol. 7, 226-236 (2009).

31. Schultheiss, $C$. et al. Next-generation sequencing of $T$ and $B$ cell receptor repertoires from COVID-19 patients showed signatures associated with severity of disease. Immunity. https://doi.org/10.1016/j.immuni.2020.06.024 (2020).

32. Ou, X. et al. Characterization of spike glycoprotein of 2019-nCoV on virus entry and its immune cross-reactivity with spike glycoprotein of SARS-CoV. Nat. Commun. https://doi.org/10.21203/rs.2.24016/v1 (2020).

(c) Open Access This article is licensed under a Creative Commons cc) Attribution 4.0 International License, which permits use, sharing, adaptation, distribution and reproduction in any medium or format, as long as you give appropriate credit to the original author(s) and the source, provide a link to the Creative Commons license, and indicate if changes were made. The images or other third party material in this article are included in the article's Creative Commons license, unless indicated otherwise in a credit line to the material. If material is not included in the article's Creative Commons license and your intended use is not permitted by statutory regulation or exceeds the permitted use, you will need to obtain permission directly from the copyright holder. To view a copy of this license, visit http://creativecommons. org/licenses/by/4.0/.

(c) The Author(s) 2020 